\title{
Demystifying White Spaces
}

\author{
Xuemin Hong ${ }^{1}$, Cheng-Xiang Wang ${ }^{1}$, John Thompson ${ }^{2}$, and Yan Zhang ${ }^{3}$ \\ ${ }^{1}$ Joint Research Institute for Signal and Image Processing, Heriot-Watt University, Edinburgh, EH14 4AS, UK. \\ ${ }^{2}$ Joint Research Institute for Signal and Image Processing, University of Edinburgh, Edinburgh, EH9 3JL, UK. \\ ${ }^{3}$ Simula Research Laboratory, 1325 Lysaker, Norway. \\ Email: xh12@hw.ac.uk, cheng-xiang.wang@hw.ac.uk, john.thompson@ed.ac.uk, yanzhang@ieee.org
}

\begin{abstract}
White spaces refer to the unused frequency voids across time or space. The vast existence of white spaces has been validated by many measurements and is widely regarded as an undesirable consequence of the fixed spectrum licensing policy. In this paper, we apply stochastic geometry to study the spatial distribution of white spaces in the presence of a random primary network with homogeneous nodes. We show that with a self-interfering constraint on the primary network, a large portion of the total area will be detected as the white space regardless of the density of the primary transmitters. Our finding suggests that the existence of white spaces is not merely a byproduct of the artificial spectrum licensing policy but also an inherent phenomenon of wireless communication systems. Two main causes of the white spaces are the self-interfering constraint and random network topology. Our model also suggests that power control and topology-aware spectrum management are the keys to eliminate white spaces and improve the overall spectrum utilization.
\end{abstract}

\section{INTRODUCTION}

The radio frequency spectrum is a scarce and precious natural resource that needs to be managed effectively. Measurements on the temporal and geographical occupations of the radio frequency revealed that only a small portion of the spectrum had been efficiently utilized [1]. Such an underutilization is commonly blamed on the fixed spectrum assignment policy, which prohibits unlicensed radio access in the licensed bands. Dynamic spectrum access (DSA) has been proposed as a means to resolve this paradox of spectrum scarcity and underutilization. It enables new wireless systems to dynamically access/share the frequency band licensed to incumbents (primary networks) on a negotiated or an opportunistic basis [2], provided that the interference to the licensee is kept insignificant.

Different DSA strategies have been proposed [2], ranging from spectrum trading/dynamic spectrum allocation that allow licensees to trade/lease their spectrum, open spectrum commons that embrace an unlicensed philosophy, to hierarchical schemes that permit secondary systems to access the spectrum of primary systems under certain interference constraints. An important type of the hierarchical DSA system is opportunistic cognitive radio networks [3]-[5] that exploit the existence of white spaces (spectrum holes) by means of radio environment sensing and adaptive transceiver reconfiguration. The white space is defined as the temporary or local frequency voids unoccupied by the primary signals. Essentially, the use of white spaces allows a secondary network to coexist with a primary network in a way that the two systems are orthogonal.
White spaces exist in both the time and space domains. Temporal white spaces come from the idle periods between busty transmissions of the primary systems. For example, [6] showed that the $802.11 \mathrm{~b}$ WLAN channel supporting a Skype conference call is idle for 89 percent of time. On the other hand, spatial white spaces occur in the "remote" areas where all the primary transmitters are sufficiently far away. A prominent example of spatial white space is the TV bands [7], [8] where the channels are interleaved so that a single subband is used in separated regions rather than the entire country. In this paper, we restrict our discussions to the spatial white spaces. The term "white space" refers to "spatial white space" in the rest of this paper.

Characterization and modeling of the white spaces are of great importance for the planning and design of opportunistic cognitive radio networks. Current understandings of the white spaces are mostly empirical and are restricted to specific bands (e.g., TV band) or measurement campaigns [1], [7], [8]. These empirical understandings fail to give a macroscopic characterization of the white spaces. Moreover, they provide little insight regarding the cause of the white spaces, whose existence is highly coupled with the characteristics of the primary network. Therefore, a theoretical model that captures the essential spatial characteristics of the white spaces is an important research subject.

White space modeling is essentially a problem of $s p a-$ tial spectrum sharing (or spatial reuse), which is a wellinvestigated subject for both cellular (planned) networks [9] and ad-hoc networks [10]. Different from previous works, in this paper we study spatial spectrum sharing with a new perspective by focusing on the area fraction of the white spaces. Moreover, we use a stochastic geometry model [11] to capture the statistical spatial properties of the white spaces. Our investigations reveal several interesting insights. First, we find that white spaces are naturally abundant in a randomized primary network. Second, power control and topology management seem to be effective tools for a cognitive (secondary) network to combat the randomness and improve the overall spectrum utilization.

The remainder of this paper is organized as follows. Section II introduces some basic stochastic geometric models, which are then applied to our system model described in Section III. In Section IV, we analyze the area fraction of the white spaces and discuss the results and their implications. Finally, conclusions are drawn in Section V. 


\section{Preliminaries in Stochastic Geometry}

Stochastic geometry has been widely used to model the nonuniformity typically observed in modern wireless networks: radio devices are not distributed uniformly and it is therefore desirable to have enough flexibility in a model to capture fluctuations in spatial diversity. In this section, we will give a brief review of some basic concepts and principles in stochastic geometry. Our review follows closely the text of Stoyan [11].

\section{A. Stationary Poisson point process}

Poisson point process is the simplest and most important random point process. It serves as the basic building block for other more complicated models. Theoretical definitions of varieties of point process frequently make reference to Poisson point processes. A random point pattern or point process $\Phi$ can be regarded as a random set $\Phi=\left\{x_{1}, x_{2}, \ldots\right\}$ where $x_{i}(1 \leq$ $i<\infty)$ is given on the $d$-dimensional (Euclidean) space $\Re^{d}$ with $\Re=(-\infty, \infty)$. In this paper we are only interested in the plenary case giving $d=2$. A stationary Poisson point process is characterized by two fundamental properties: (1) Poisson distribution of point counts, the number of points of $\Phi$ in a bounded area $A$ has a Poisson distribution of mean $\lambda A$ for some constant $\lambda$; (2) Independent scattering, the number of points of $\Phi$ in $k$ disjoint areas form $k$ independent random variables. The parameter $\lambda$ occurring in property (1) is called the intensity or density and it gives the mean number of points to be founded in a unit area. Property (2) is also known as the "completely random" or "purely random" property.

\section{B. Thinning}

There are three fundamental operations which produce new point processes from old ones: thinning, clustering, and superposition. Here we will review the thinning operations only. A thinning operation uses some definite rule to delete points of a point process $\Phi$, thus yielding the thinned point process $\Phi_{t h}$. The simplest thinning is $p$-thinning. In this each point of $\Phi$ has probability $1-p$ of suffering deletion, and its deletion is independent both of the locations and of the possible deletions of any other points of $\Phi$. Consequently, $p$-thinning belongs to a class of thinning operations called independent thinnings, which means there is no interaction between the points, so that the thinning functions (which are independent of $\Phi$ ) determine the operation completely. A generalization allows dependence on the configuration of $\Phi$, giving the class of dependent thinnings. The Matern hard-core point process described in the following sub-section is a point process resulting from dependent thinning.

\section{Matern hard-core point process}

A hard-core point process is a point process in which the constituent points are forbidden to lie closer together than a certain minimum distance, denoted as $d_{\min }$. These hard-core models serve to describe patterns produced by the locations of centers of non-overlapping circles of radius $r=d_{\min } / 2$. Here we will describe a model called the Matern hard-core process, which yields a higher eventual density of points among some other proposed hard-core process models [11]. The Matern hard-core process is essentially a dependent thinning applied to $\Phi$, a stationary Poisson process of density $\lambda$. The points of $\Phi$ are marked independently random numbers uniformly distributed over $(0,1)$. The dependent thinning retains the point $x$ of $\Phi$ (with mark $m(x)$ ) if the circle $C\left(x, d_{\min }\right.$ ) contains no points of $\Phi$ with marks smaller than $m(x)$. Formally the thinning process $\Phi_{t h}$ is given by [11]

$\Phi_{t h}=\left\{x \in \Phi: m(x)<m(y)\right.$ for all $y$ in $\left.\Phi \cap C\left(x, d_{\min }\right)\right\}$.

The density $\lambda_{t h}$ of $\Phi_{t h}$ can be obtained as [11]

$$
\lambda_{t h}=\frac{1-\exp \left(-\lambda \pi d_{\min }^{2}\right)}{\pi d_{\min }^{2}} .
$$

Given $d_{\min }$, the highest density yield by a Matern hard-core process is then given by

$$
\lambda_{t h}^{\max }=\lim _{\lambda \rightarrow \infty}\left(\lambda_{t h}\right)=\frac{1}{\pi d_{\min }^{2}}
$$

We note that this density does not represent the closest possible packing of the circles. In the closest packing, the centers of the circles forms a regular cellular-like structure and we have the point density $\lambda_{\text {cell }}=2 /\left(\sqrt{3} d_{\min }^{2}\right)$.

\section{Germ-grain models}

Germ-grain models is an important example of a random set models. Given a marked point process $\Psi=\left\{\left[x_{n} ; \Xi_{n}\right]\right\}$ where the $x_{n}$ are points of $\Re^{d}$ and the $\Xi_{n}$ are compact subsets of $\Re^{d}$, a germ-grain model $\Xi$ is defined based on the point process as the union given by [11]

$$
\Xi=\cup_{n=1}^{\infty}\left(\Xi_{n}+x_{n}\right)
$$

where the $x_{n}$ are the germs and the $\Xi_{n}$ are the primary grains of the germ-grain model. In the case when $\Xi$ is stationary it is useful to introduce the 'typical' grain $\Xi_{0}$, which is a random closed set of the same distribution as the sets in the sequence of primary grains $\Xi_{n}$ but independent from $\Xi_{n}$ and the germs. An important measure of the germ-grain model is the volume faction $\chi$, defined as the mean fraction of volume occupied by $\Xi$ in a region of unit volume. When the grains fails to overlap with probability one and $\Xi$ is stationary, then the volume fraction is given by [11]

$$
\chi=\lambda \bar{V},
$$

where $\lambda$ is the intensity of the germ process $\Psi$ and $\bar{V}$ is the mean volume of a typical grain $\Xi_{0}$.

\section{SYSTEM MODEL}

We consider a random primary network on a plane operating on a specific frequency band. The spatial distribution of the white spaces ultimately depends upon the behaviors of the primary transmitters, e.g., their locations and transmit powers. Based on the locally perceived primary signal power level, a point on the plane can be marked as either a black space, grey space, or white space. The black spaces refer to spectra 
occupied by high power primary signals, while the grey spaces refer to spectra occupied by low power primary signals. The essence of the transmitter behaviors in a typical modern wireless network can be captured by the following stochastic models.

\section{A. User distribution as Poisson point process}

The locations of potential primary transmitters are modeled as a stationary Poisson point process $\Phi$ of intensity $\lambda$. Such a distribution for radios is widely used in the literature [12], [13] For ad-hoc or mobile networks, this stationary Poisson point process model is usually justified. Even for planned networks (e.g., cellular network), a Poisson point pattern can still be representative since the constraints (cost, building heights) on the locations of base stations lead to an effective randomness of the transmitter distribution [14].

\section{B. Active transmitter distribution as Matern hard-core point process}

To avoid/mitigate self-interference, a subset of active transmitters are selected among all the potential transmitters so that every two active transmitters are spatially separated from each other by at least a distance $d_{\text {min }}$. Such a selection process, enabled in practice by channel contention/allocation protocols, is typically randomized to arbitrate the accesses of all the potential primary transmitters. This random channel contention can be mathematically treated as a dependent thinning operation and the resulting set of active transmitters can be modeled as Matern hard-core point processes $\Phi_{t h}$.

\section{Radio propagation as germ-grain models}

A propagation model is defined as a closed-set $\Xi_{n}$ that characterizes the areas of black/grey space around a transmitter. Such a propagation model can be either deterministic (pathloss-only channels) or random (fading channels). Using the Matern hard-core processes $\Phi_{t h}$ as the germs and the propagation models as the grains, we can adopt the germ-grain model $\Xi$ to represent the total areas of black/grey spaces. The complementary set of $\Xi$, denoted as $\bar{\Xi}$, characterizes the white space areas. The use of such a germ-grain model implies that a black/grey space is uniquely defined by a single transmitter. In other words, we neglect the case where accumulating powers from different transmitters give birth to new black/grey spaces.

In this paper we use two simple deterministic propagation models. Both models belong to the category of protocol mod$e l s$ and use the following assumptions: (1) uniform power: the transmit powers of all active nodes are identical; (2) uniform transmission and propagation environment: only the pathloss is considered and the signal emitted by every transmitter presents the same pathloss characteristic. In our first model, called "black-white model", we only distinguish between the black and white space. The grain $\Xi$ (for black space) is given as a circular disk of radius $r$, where $r$ denotes the transmission range. This simple model is useful in revealing interesting insights without over-complicating the problem. The second propagation model we consider is called the "black-greywhite" model since it further distinguishes the grey space. A new parameter $R$ is incorporated into this model to denote the interfering range of an active primary transmitter. As shown in Fig. 1, the black space area is still the smaller disk of radius $r$, while the grey space area is given by the concentric disk of radius $R$ excluding the areas of black spaces.

\section{Area Fractions of White Spaces}

The properties of random sets can be characterized by various random measures [11]. In this paper we are interested in the first moment measure on a plane, i.e., the area fraction.

\section{A. Area fraction with black-white propagation model}

We consider the area fraction $\chi_{b}$ of the black spaces keeping in mind that the area fraction of the white spaces is simply $\chi_{w}=1-\chi_{b}$. With the black-white propagation model, the minimum distance $d_{\text {min }}$ of two non-interfering transmitters is $d_{\min }=2 r$, where $r$ is the transmission range of an active transmitter. Given $d_{\mathrm{min}}$, the density of active transmitters $\lambda_{m}$ can be computed from (2). Moreover, since there is no overlapping between two arbitrary disks of black space, (5) can be directly applied to calculate the area fraction of the black spaces, i.e.,

$$
\chi_{b}=\lambda_{t h} \pi r^{2}=\frac{1-\exp \left(-4 \lambda \pi r^{2}\right)}{4}
$$

where $\lambda$ is the intensity of the primary transmitters. From (6) we can see that $\chi_{b}<1 / 4$ and thus $\chi_{w}>3 / 4$. In other words, regardless of $\lambda$ and $r$, at least 75 percent of the plane will be detected as the white space. This finding is somewhat surprising since one would generally expect little existence of the white space when the primary node density $\lambda$ is large enough.

The above bound on $\chi_{b}$ mainly results from the Matern hard-core point process, which itself has a bound on the maximum density of active transmitters given by (3). We note that there are two basic properties of a Matern hard-core point process. The first one is the minimum distance requirement. Without such a requirement we can use stationary Poisson point processes as the germs and the general germ-grain model is reduced to a boolean model [11]. The corresponding area fraction of a boolean model is given by [11]

$$
\chi_{b}^{b o o l}=1-\exp \left\{-\lambda \pi r^{2}\right\} .
$$

Obviously, in this case the white space extincts when $\lambda$ tends to infinity. The second basic property of the Matern hard-core point process is its "randomness". This randomness is rooted in the purely random property [11] of the original Poisson point process and the randomized dependent-thinning process used in the construction of a Matern hard-core process (see Section II.C). Without this randomness requirement we can closely place non-overlapping circles into a regular cellular structure, which gives an area fraction $\chi_{b}^{\text {cell }}=\pi /(2 \sqrt{3})$.

Unfortunately, both the above two requirements are common in modern wireless networks: the spatial separation comes 
from self-interference constraints and the randomness comes from the mobility of the nodes. This leads us to the following remark: White space is not merely an artificial by-product of spectrum licensing but to certain extent an inherent nature of wireless communications.

A question followed naturally is: how can we "eliminate/reduce" the white space to improve the spectrum utilization? To this end, we assume an opportunistic cognitive radio network coexisting with the primary network on a noninterfering basis. If the cognitive radio tranmitters have the same characteristics as the primary transmitters (i.e., the same "grains"), their presences would be the same as increasing the total primary transmitter density $\lambda$. In this case the $1 / 4$ bound on $\chi_{b}$ still applies, which means that even in the presence of a secondary network we still have relatively low (spatial) spectrum utilization efficiency. From this discussion we conclude the following remark: To effectively improve the spectrum utilization, it is desirable to differentiate the transmission characteristics of the secondary (cognitive) devices from the primary transmitters.

In addition, we can image that if the cognitive radios are able to adjust their transmit powers and thereby adaptively change their coverage areas to fill in the spatial voids, the overall spectrum utilization can be significantly improved.

\section{B. Area fraction with black-grey-white propagation model}

In this subsection we consider a more realistic propagation model: the black-grey-white model. Again we will first work on the area fraction $\chi_{b g}$ of the black/grey spaces and then get the white space area fraction as $\chi_{w}=1-\chi_{b g}$. As illustrated in Fig. 1, given the transmission range $r$ and interfering range $R$ $(R>r)$, the minimum distance between two non-interfering primary transmitters is $d_{\text {min }}=r+R$. We consider a germgrain model $\Xi$ where the germs are the Matern hard-core processes $\Phi_{t h}$ with a minimum separation of $d_{\min }$ and the grains are identical disks of radius $R$. We aim to obtain the area fraction $\chi_{b g}$ of $\Xi$. It turns out that (5) can no longer be used to calculate the area fraction since grains can now overlap. Nevertheless, we can still evaluate the lower and higher bounds of $\chi_{b g}$ as follows.

1) Lower bound of $\chi_{w}$ : The lower bound of $\chi_{w}$ corresponds to the upper bound of $\chi_{b g}$. Neglecting the overlapping effects of the grains of $\Xi$ and assuming every grain has an "effective" area of $\pi R^{2}$, an (loose) upper bound of $\chi_{b g}$ can be obtained as

$$
\chi_{b g}^{U}=\frac{R^{2}}{(R+r)^{2}}\left(1-\exp \left\{-\lambda \pi(R+r)^{2}\right\}\right)
$$

The lower bound $\chi_{w}^{L}$ of $\chi_{w}$ is then given by $\chi_{w}^{L}=1-\chi_{b g}^{U}$.

2) Upper bound of $\chi_{w}$ : We consider two lower bounds of $\chi_{b g}$. For the first one, we assume a new germ-grain process $\Pi_{1}$ where the germs are still given by $\Phi_{t h}$ but the grains are now disks of radius $\frac{R+r}{2}$. Clearly, the area fraction $\chi_{b g}^{L 1}$ of $\Pi_{1}$ serves as a lower bound of $\chi_{b g}$. Since the grains of $\Pi_{1}$ fail to overlap, from (6) we have

$$
\chi_{b g}^{L 1}=\frac{1-\exp \left(-\lambda \pi(R+r)^{2}\right)}{4} .
$$

The second lower bound can be obtained with the assistance of a new germ-grain process $\Pi_{2}$ where the germs are now stationary Poisson point processes with intensity $\lambda_{t h}$ and the grains remain as disks of radius $R$. In this case $\Pi_{2}$ is reduced to a boolean model [11]. From (7), the area fraction $\chi_{b g}^{L 2}$ of $\Pi_{2}$ can be obtained as

$$
\begin{aligned}
\chi_{b g}^{L 2} & =1-\exp \left\{-\lambda_{t h} \pi R^{2}\right\} \\
& =1-\exp \left\{-\frac{R^{2}}{R^{2}+r^{2}}\left(1-\exp \left\{-\lambda \pi(R+r)^{2}\right\} .\right)\right\}
\end{aligned}
$$

To prove that $\chi_{b g}^{L 2}$ serves as a lower bound, we first introduce another germ-grain process $\Pi_{0}$ where the germs are stationary Poisson point process with intensity $\lambda$ and the grains are disks of radius $R$. It turns out that both $\Xi$ and $\Pi_{2}$ can be generated from $\Pi_{0}$ by thinning the germs. A dependent Matern hard-core $t$-thinning [11] is used to generate $\Xi$ while an independent $p$-thinning is used to generate $\Pi_{2}$. When a germ is deleted, the corresponding effective grain (the area that is not overlapped with other grains) is also deleted. The fact that the Matern hard-core $t$-thinning tends to delete closely spaced germs can be translated as that it tends to delete germs with less effective grains. Moreover, both thinning operations have the same retaining probability, i.e., statistically both thinning operations delete the same number of germs. Consequently, the overall effect would be that the $p$-thinning deletes more effective grains from $\Pi_{0}$ compared with the Matern hard-core $t$-thinning. It follows that the area faction of $\Pi_{2}$ would be smaller than that of $\Xi$, so $\chi_{b g}^{L 2}$ is a lower bound of $\chi_{b g}$. It is easy to prove that $\chi_{b g}^{L 2}$ is a tighter lower bound, i.e., $\chi_{b g}^{L 2}>\chi_{b g}^{L 1}$. Therefore, the upper bound of the white space area fraction can be evaluated as $\chi_{w}^{U}=1-\chi_{b g}^{L 2}$.

\section{Numerical results}

In this sub-section, the bounds of $\chi_{w}$ given by (8) and (10) under the black-grey-white propagation models will be evaluated numerically. In Fig. 2, we set the transmitter density $\lambda=0.001$ and show the bounds from as functions of the transmission range $r$ with different $R / r$ ratios. We see that $\chi_{w}$ decreases with increasing $R / r$ ratios. This can be explained intuitively: a larger $R / r$ ratio means a wider protection zone of the primary services and therefore the amount of white spaces reduces. Moreover, Fig. 2 shows that white spaces contribute to a significant portion of the total area. When $r \rightarrow \infty$, the upper and lower bounds of $\chi_{w}$ converges to $1-\exp \left(-\frac{R^{2}}{(R+r)^{2}}\right)$ and $\frac{R^{2}}{(R+r)^{2}}$, respectively.

In Fig. 3, we fixed $R / r=3$ (as the primary service protection requirement) and show the bounds as functions of the transmitter density $\lambda$ with different values of $r$. We can see that given $r$, the area fraction of the white spaces reduces gradually with increasing $\lambda$. On the other hand, given $\lambda$, the white space area fraction reduces with increasing $r$. With large values of $r$ or $\lambda$, the bounds converge as discussed previously.

\section{Conclusions}

In this paper, the spatial distributions of white spaces have been studied using stochastic geometry. We have considered 
a primary network with homogeneous transmitters whose distributions on a plane are modeled as stationary Poisson point processes. We have modeled the areas of black/grey spaces as random closed sets generated from germ-grain models, where the germs (locations of active transmitter) are Matern hard-core point processes and the grains are given by two deterministic propagation models. With such a germgrain model, the area fractions of the white space have been studied. We have found that a large amount of white spaces exists, which can be explained by the randomness and transmitter separation requirements of the primary network. We conclude that white space is a natural by-product of many wireless systems. To effectively exploit the white space, it is desirable for a cognitive radio network to implement adaptive technologies such as power control or topology-aware channel contention protocols.

\section{REFERENCES}

[1] Federal Communication Commission, Facilitating opportunities for flexible, efficient, and reliable spectrum use employing cognitive radio technologies, NPRM \& Order, ET Docket No. 03-108, FCC 03-322, 30 Dec. 2003.

[2] Q. Zhao and B. M. Sadler, "A survey of dynamic spectrum access," IEEE Signal Processing Mag., vol. 24, no. 3, pp. 79-89, May 2007.

[3] H. H. Chen, and M. Guizani, Next Generation Wireless Systems and Networks, Chichester: John Wiley \& Sons, 2006.

[4] S. Haykin, "Cognitive radio: brain-empowered wireless communications", IEEE J. Selected Areas Commun., vol. 23, no. 2, pp. 201-220, Feb. 2005.

[5] I. F. Akyildiz, W. Y. Lee, M. C. Vuran and S. Mohanty, "NeXt generation/dynamic spectrum access/cognitive radio wireless networks: a survey", Computer Networks, vol. 50, no. 13, pp. 2127-2159, Sept. 2006.

[6] S. Geirhofer, L. Tong, and B.M. Sadler, "Dynamic spectrum access in the time Domain: modeling and exploiting white space", IEEE Commun. Mag., vol. 45, no. 5, pp. 66-72, May 2207.

[7] S. Sengupta, S. Brahma, M. Chatterjee, and S. Shankar N, "Enhancements to cognitive radio based IEEE 802.22 air-interface", Proc. IEEE ICC'07, Glasgow, UK, June 2007, pp. 5155-5160.

[8] V.R., Petty, e.t.c., "Feasibility of Dynamic Spectrum Access in Underutilized Television Bands", IEEE Proc. DySPAN'07, Dublin, Ireland, Apr. 2007, pp. 331-339.

[9] I. Katzela and M. Naghshineh, "Channel assignment schemes for cellular mobile telecommunication systems: a comprehensive survey", IEEE Personal Commun., vol. 3, no. 3, pp. 10-31, June 1996.

[10] X. Guo, S. Roy, and W.S. Conner, "Spatial reuse in wireless ad-hoc networks", Proc. IEEE VTC'03-Fall, Florida, USA, Oct. 2003, pp. 1437 1442.

[11] D. Stoyan, W.S. Kendall, and J. Mecke, Stochastic Geometry and its Applications, Chichester: John Wiley \& Sons, 1986.

[12] E. S. Sousa, "Performance of a spread spectrum packet radio network link in a poisson field of interferers," IEEE Trans. Infor. Theo., vol. 38, no. 6, pp. 1743-1754, Nov. 1992.

[13] R. Menon, R. M. Buehrer, and J. H. Reed, "Impact of exclusion region and spreading in spectrum-sharing ad hoc networks", Proc. First Int. Workshop on Techno. and Policy for Accessing Spectrum, Boston, USA, Aug. 2006, article no. 7.

[14] F. Baccelli, M. Klein, M. Lebourges, and S.Zuyev, "Stochastic geometry and architecture of communication networks", J. Telecommun. Systems vol.7, pp. 209-227, 1997.

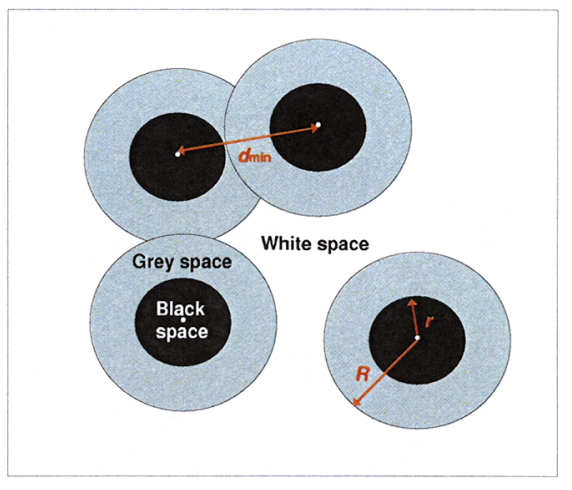

Fig. 1. System model: a plane is divided into black spaces, grey spaces, and white spaces by randomly distributed homogeneous primary transmitters

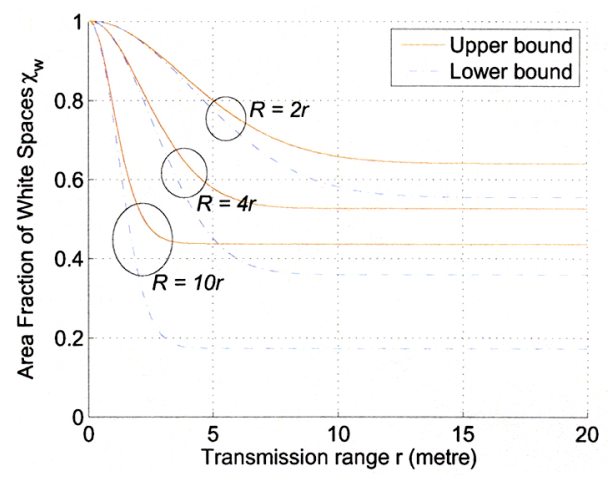

Fig. 2. Upper and lower bounds of the white space area fraction $\chi_{w}$ as functions of $r$ with different values of $R / r(\lambda=0.001)$.

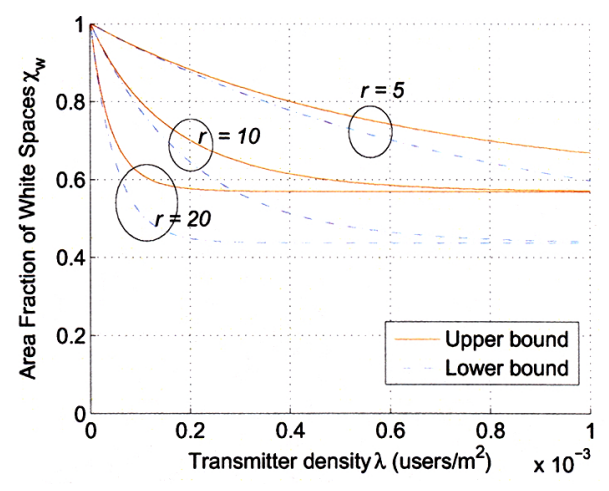

Fig. 3. Upper and lower bounds of the white space area fraction $\chi_{w}$ as functions of $\lambda$ with different values of $r(R / r=3)$. 